\title{
Ma questa Geisha è giapponese, italiana o inglese?
}

\author{
Paolo D'Achille
}

PUBBLICATO: 14 GIUGNO 2019

\section{Quesito:}

Sono pervenute alcune domande sulla corretta grafia italiana della parola geisha, sulla forma del plurale e sulla possibile omissione dell'articolo prima del termine.

\section{Ma questa Geisha è giapponese, italiana o inglese?}

I 1 termine geisha (tratteremo più oltre di questa grafia) è un nipponismo, cioè una parola che proviene dalla lingua giapponese. I nipponismi costituiscono una quota abbastanza rilevante tra gli esotismi entrati in italiano e il loro ingresso è stato mediato prima dal francese e poi dall'inglese. Ricordiamo nomi di sport, come il judo e il karate (queste le grafie e le pronunce oggi prevalenti; in passato si usava accentare i termini sull'ultima vocale, judó e karaté, per rendere l'accentazione propria del francese), di attività artistiche, come origamie ikebana, di capi d'abbigliamento, come il kimono (anche nell'adattamento chimono), e ancora voci come harakiri (la grafia karakiri sarebbe scorretta), kamikaze (usata poi prevalentemente con riferimento al terrorismo di matrice islamica), il recente karaoke, il recentissimo sudoku e, infine, tsunami, voce che dopo il tremendo maremoto della fine del 2004 viene spesso usata col valore figurato di 'ondata'.

Dalla lingua giapponese vengono poi anche parole che si riferiscono specificamente alla cultura locale, come samurai e come, appunto, geisha, che significa "in Giappone, giovane donna istruita nella musica, nella danza e nell'arte del tè, addetta a intrattenere gli uomini ospiti di conviti privati o pubblici" (GRADIT, dove si segnala che geisha è una parola composta da gei 'arte, artistico' e sha 'persona', che dunque, propriamente, significa 'persona versata per le arti'). In senso estensivo, la parola può essere usata per "donna di facili costumi; cortigiana" (anche questa definizione è del GRADIT). Ma direi che ormai quest'accezione è diventata piuttosto rara: geisha oggi si usa piuttosto, in senso erotico, in espressioni come "sarò la tua geisha", "sarai la mia geisha" (abbastanza diffuse in rete), che non implicano affatto (o almeno non necessariamente) il mercimonio.

Ancora secondo il GRADIT, che riprende evidentemente un saggio di Marco Mancini, la prima attestazione del termine risalirebbe av. 1557 [recte ${ }_{1547}$ ], in uno dei testi compresi nella raccolta Navigationi e viaggi di G.B. Ramusio. Va peró precisato che la parola compare qui nella grafia portoghese goxo e per di più in contesti in cui il riferimento al nostro designatum è tutt'altro che sicuro. In ogni caso, si tratta di un'attestazione isolata, con soluzione di continuità rispetto a quelle posteriori.

La parola iniziò davvero a circolare in Italia solo alla fine dell'Ottocento, quando in tutta Europa si ebbe un'ondata di gusto orientaleggiante che penetrò anche nella letteratura, nell'arte, nella musica e che mise in circolazione oggetti, tradizioni, usi e costumi provenienti dall'Estremo Oriente, diffondendo, insieme alle cose, anche le corrispondenti parole. Ecco cosi che la nostra geisha entra stabilmente in italiano, prima nella grafia (forse spagnoleggiante) guesha, in D'Annunzio (I884), quindi in quella (certamente francesizzante) di guecha, attestata in un articolo apparso in "La scienza per tutti. Giornale popolare illustrato" del I894 (pp. I88-I89), o guècha, come si legge nel libretto, di Luigi Illica, dell'Iris di Pietro Mascagni (I898). La protagonista di quest'opera è una ingenua musmé, che 
viene rapita dal nobile Osaka, il quale ben presto l'abbandona; esposta in un quartiere malfamato e maledetta dal padre, preferisce togliersi la vita e viene alla fine trasformata nell'omonimo fiore. Ho riportato la trama dell'Iris perché, forse proprio in rapporto ad essa, e nonostante la purezza del personaggio femminile, anche il nipponismo musmé, qui attestato come mousmé (il GRADIT lo registra invece come musume), pur significando semplicemente, in giapponese, 'giovane donna', è stato usato in italiano come sinonimo di geisha e ha quindi assunto, ancora più indebitamente di questa parola, il senso di 'prostituta'.

Ma torniamo alla nostra geisha. Nella Madama Butterfly di Giacomo Puccini (I9oi), su libretto ancora di Illica e di Giuseppe Giacosa, il termine compare due volte nella forma adattata ghescia (almeno nel libretto originale e nelle edizioni più attendibili; nei sottotitoli televisivi mi è capitato di leggerlo nella grafia oggi prevalente, mentre in rete si trova anche riprodotto come ghesha). Ecco i passi: "conobbi la ricchezza. Ma il turbine rovescia / le quercie più robuste... e abbiam fatto la ghescia / per sostentarci", dice la protagonista Cio-Cio-San nel primo atto; e nel secondo: "E Butterfly, orribile destino, / danzerà per te! / E come fece già / la ghescia canterà". Questa è del resto la forma della prima sicura attestazione italiana della parola, che ha numerose occorrenze (come singolare o come plurale invariabile) in un volume di Pietro Savio, Il Giappone al giorno d'oggi nella sua vita pubblica e privata,politica e commerciale (Milano, Treves, I876):

Ghescia o ballerina (p. 22; nel sommario del capitolo I).

Ma se sgradita ci torna la musica, non si può dire lo stesso della danza, che colle sue pose molli e graziose rapisce pur anche i nostri stranieri. Essa è soltanto eseguita da giovani donne, a cui si dà il nome di ghescia, e le quali esprimono sempre scene di passione che trasportano gli uomini che stanno contemplandole (ivi, p. 40).

Ognuna di esse porta una compagnia d'amici che vi cenano allegramente, esaltandosi con liquori e colla musica delle giovine [sic] ed amabili ghescia (suonatrici) che stanno con loro (ivi, p. 198).

Anche il Yankiro, quartiere appartato dei sollazzi posto in Takascima-ciò è soggetto a tassa e produce più di quattromila yen all'anno, e mille altri yen sono prodotte [sic] da quella sulle ghescia(suonatrici) che colà dimorano per dilettare col canto e col suono il pubblico (ivi, p. 2r2).

Risale al 1905 la registrazione nel Dizionario moderno di Alfredo Panzini della forma ghèiscia, che è quella scelta come lemma principale nel GDLI e che è segnalata come variante secondaria di geisha dallo Zingarelli 2019 (che continua a datare, tardivamente, il termine a quell'anno, in base al DELI), mentre la diffusione (e non solo in Italia) della grafia anglicizzante geisha sembra dovuto al successo internazionale dell'operetta La Geisha di Sidney Jones (libretto di Howen Halle), del ı896, la cui prima traduzione italiana, risalente già al ı́o, mantenne il titolo nella veste inglese.

Ma la parola, in questa stessa forma (scritta ora in tondo ora in corsivo, come plurale invariabile), era già comparsa sette anni prima in un articolo della "Nuova Antologia":

Furono scostati i lumi dal pavimento, ed il padron di casa esclamò: Geisha!...geisha!... Una delle principali attrattive ci era offerta. Non vi è, di fatti, pranzo di giapponese, per povero che sia, nel quale si lascino gli invitati senza le danze, necessario compimento per ogni festa (Giovanni De Riseis, Feste giapponesi. Ricordi di viaggio, in "Nuova Antologia", s. 3ª , vol. LIII, I5 settembre I894, pp. 294-316, a p. 300).

Preparata così la messa in scena, entran subito in azione le tre geisha, con passi studiati (ivi); 
nell'agitarsi di quei sapienti ventagli; nei piccoli gridi, nelle occhiate languide delle geisha v'era tanto pei giapponesi da rimanere come ipnotizzati, presi da un fascino strano... (ivi, p. 302);

e, singolare riunione di cose prosaiche e poetiche, le geisha ricominciavano il loro geniale trattenimento (ivi, p. 303).

La lessicografia italiana (ma anche quella francese) registra ormai la parola solo nella grafia geisha, che è di fatto l'unica oggi diffusa, ma che, come giustamente rilevano i nostri lettori, non rende appieno la pronuncia. Tuttavia, se è vero che la $g$ prima di e per essere pronunciata come velare richiederebbe l'h, va detto che la successiva presenza di sh ci assicura che si tratta di una parola straniera e non italiana. D'altra parte, la proposta avanzata da un lettore di scrivere gheisha mi pare incoerente: o si opta per l'italianizzazione totale o per la riproduzione della grafia inglese. Ricordo tuttavia che, oltre che in alcune riproduzioni in rete del libretto della Madama Butterfly, Ghesha e, al plurale, Gheshas sono le forme attestate nella prima traduzione italiana del romanzo Madame Chrisanthéme di Pierre Loti, che usci in Francia nel I887 e che certo ha contribuito anch'esso alla fortuna del termine (La Signora dei Crisantemi. Romanzo di Pierre Loti. Prima versione italiana, Milano, Società Editoriale Milanese, I9o8, pp. 35-36; nell'originale francese la grafia è guécha).

La percezione della sostanziale estraneità della parola alla nostra lingua determina qualche difficoltà nella resa del plurale. L'adattamento ghescia, pur usato come invariabile (lo si è visto) da Savio nel I876 e in altri esempi di poco posteriori, ha il regolare plurale ghesce (usato per es. da Luigi Barzini, che lo mette in corsivo: "Non avevano la grazia delle ghesce, quelle piccole cinesi coperte di strani gioielli, infagottate di damaschi, imbellettate come bambole"; L. Barzini, Qua e là per il mondo. Racconti e ricordi, Milano, Hoepli, I9I6, p. 243), cosi come gheiscia ha il plurale gheisce, che ha numerose attestazioni nel corso del tempo e che è documentato già nel I905, in corsivo, in questo passo (dove tuttavia viene riferito alle danze delle geishe):

e furono suonate musiche antiche e danzate delle gheisce, danze tradizionali (Il Mikado, le sue abitudini, la sua famiglia, in "La Lettura", V, 1905, pp. 376-380, a p. 379).

Nel caso della forma geisha possiamo avere sia il "normale" geishe (che è il plurale indicato dal GRADIT e che è quello che anch'io consiglio di usare, senza dover ricorrere al carattere corsivo), sia geishas, con l'aggiunta della -s che si avrebbe in inglese (segnalo che la -s si trova anche in guéchas, nel libretto dell'Iris, nelle Ghescias della traduzione di Loti e che ho letto perfino un plurale Gheiscias con riferimento al coro delle amiche della protagonista della Madama Butterfy, che nel primo atto fanno da corteo nuziale), sia il trattamento (documentato, come si è visto, già nel I894) di geisha come invariabile, che pare in sintonia con una certa tendenza all'invariabilità propria dell'italiano contemporaneo. Dunque può capitare di imbattersi in grafie come le geishe, le geishas e anche le geisha. Una situazione analoga, del resto, si ha anche per l'ispanismo telenovela, il cui plurale oscilla tra telenovele, telenovelas e telenovela.

Quanto allutilizzazione della forma senza articolo, che viene segnalata come "scimmiottamento" dell'inglese da un nostro lettore (ma perché? Tutti o quasi tutti i sostantivi italiani possono essere usati privi di articolo in determinati contesti), l'ho trovata di recente nel titolo della mostra Geisha l'arte e la persona tenutasi a Roma nel settembre 20r8, dove si spiega appunto con la collocazione della parola in un titolo, all'interno del quale il fascino esotico di (o della?) geisha viene certamente accresciuto dalla mancata anteposizione di un articolo "nostrano". 
Nota bibliografica:

- Marco Mancini, Voci orientali ed esotiche nella lingua italiana, in Storia della lingua italiana, a cura di Luca Serianni, Pietro Trifone, vol. III, Le altre lingue,Torino, Einaudi, 1994, pp. 825-279.

- Paolo D'Achille, Prime apparizioni di ideofoni ed esotismi in libretti d'opera, in "Lingua nostra", LVII, i996, pp. I-6.

- Chiara Coluccia, Il prestito linguistico nella lessicografia contemporanea: nipponismi e sinismi nei vocabolari, in Etimologia e storia di parole. Atti del XII Convegno ASLI (Firenze, Accademia della Crusca, 3-5 novembre 2016), a cura di Luca D'Onghia e Lorenzo Tomasin, Firenze, Franco Cesati, 2018, pp. 207-218.

\section{Cita come:}

Paolo D'Achille, Ma questa Geisha è giapponese, italiana o inglese?, "Italiano digitale", 2019, IX, 2019/2 (aprile-giugno)

DOI: $10.35948 / 2532-9006 / 2020.3124$

Copyright 2019 Accademia della Crusca

Pubblicato con licenza creative commons CC BY-NC-ND 